\title{
THE CONTACT BINARY AE PHOENICIS - AN ANALYSIS FROM DECONVOLUTED SPECTRA*
}

\author{
H. J. BECKER \\ Universitäts-Sternwarte Bonn, F.R.G.
}

(Received 30 August, 1983)

\begin{abstract}
A method is described for an analysis of deconvoluted stellar spectra, and applied to new spectroscopic observations of the close eclipsing system AE Phuenicis, obtained with the $1.52 \mathrm{~m}$ telescope of the Southern European Observatory at La Silla, Chile. Results are presented for the rotational velocities of this variable, together with a proposed model of the system.
\end{abstract}

60 spectrograms of the W UMa-type eclipsing binary AE Phe were obtained with the coudé spectrograph at the $1.52 \mathrm{~m}$ telescope of the European Southern Observatory, $\mathrm{La}$ Silla, Chile. The emulsion is IIa- 0 , the dispersion $20 \AA \mathrm{mm}^{-1}$. The spectrograms were digitized with the PDS 1010A microdensitometer of the Astronomical Institutes, University of Bonn.

We observe the intensity distribution

$$
I(\lambda)=A(\lambda) * B(\lambda) * C(\lambda),
$$

where $A(\lambda)$ is the original spectrum without rotational broadening; $B(\lambda)$ is the instrument profile; $C(\lambda)$ is the broadening function due to rotation. The symbol $*$ denotes convolution. This assumes that the usual 'uniform profile approximation' is justified.

The broadening function $C(\lambda)$ can be derived from the observed $I(\lambda)$ by deconvolution. For its derivation, we make use of the convolution theorem

$$
\tilde{I}(v)=\tilde{A}(v) \tilde{B}(v) \tilde{C}(v) \text {. }
$$

Furthermore, we can use a standard spectrum of the same spectral type ( $\kappa$ Cet, $v \sin i=0 \mathrm{~km} \mathrm{~s}^{-1}$ ) for $A(\lambda)$. For $\kappa$ Cet, we have

$$
D(\lambda)=A(\lambda) * B(\lambda),
$$

where $B(\lambda)$ is the same instrumental profile as above. Then,

$$
\tilde{I}(v)=\tilde{D}(v) \tilde{C}(v) \text { or } \tilde{C}(v)=\tilde{I}(v) / \tilde{D}(v),
$$

i.e., division of the Fourier transforms. Transforming the result back into the spatial domain yields $C(\lambda)$. Before transforming back, a filter must be applied to suppress high Fourier frequencies (noise).

\footnotetext{
* Paper presented at the Lembang-Bamberg IAU Colloquium No. 80 on 'Double Stars: Physical Properties and Generic Relations', held at Bandung, Indonesia, 3-7 June, 1983.
} 
The practical procedure is as follows:

- digitization of the spectra of the standard star ( $\kappa$ Cet);

- digitization of the spectra of the program star (AE Phe);

- conversion from $x$ to $\lambda$ (dispersion curve), then to $\log \lambda$ (the radial velocity introduces a constant shift for the complete data interval);

- determination of the $\lambda$-dependent characteristic curve and conversion density $\rightarrow$ intensity;

- FFT after usual treatment of data arrays;

- division, filter function;

- FFT.

We have investigated the two spectral intervals $\lambda \lambda 4000 \ldots 4080$ (mainly Fe I lines) and $\lambda \lambda 4080 \ldots 4120(\mathrm{H} \delta)$. Results are shown in Figures 1 to 6.

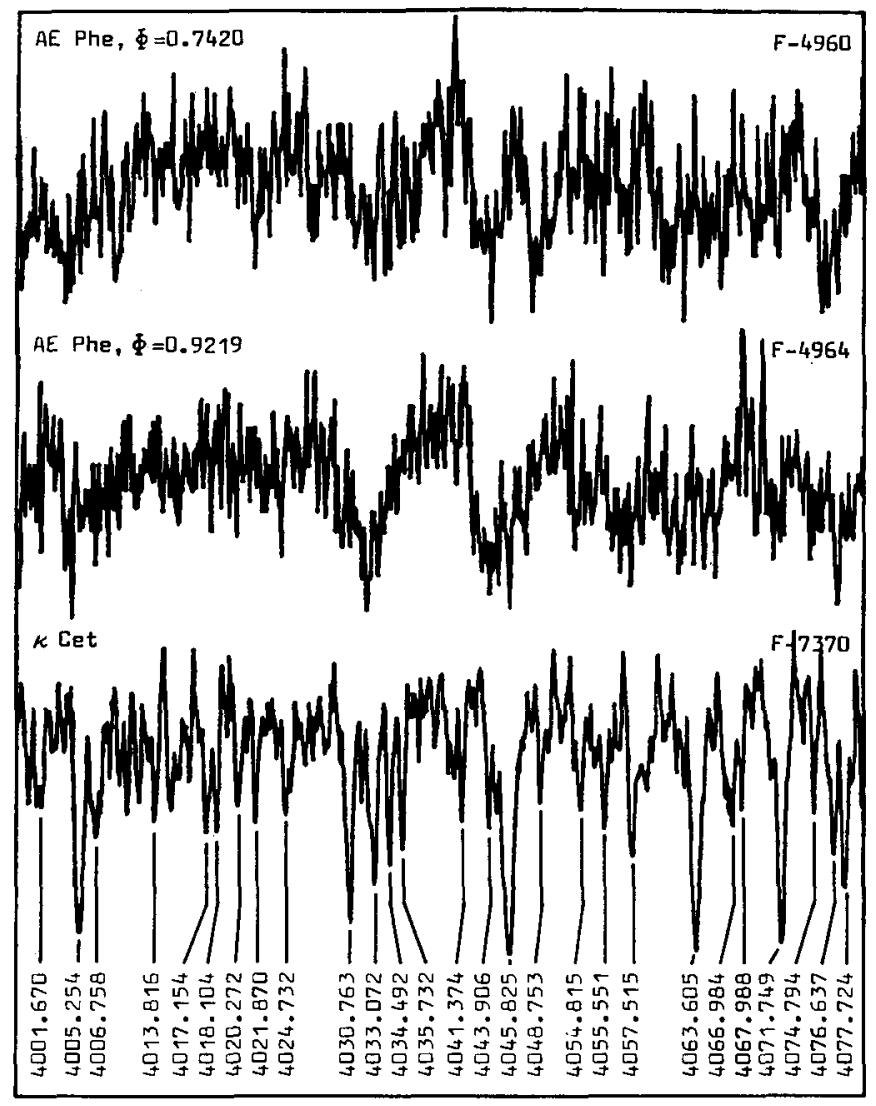

Fig. 1. $I(\lambda)$ in the interval $\lambda \lambda 4000 \ldots 4080$, AE Phe at different phases, and $\kappa$ Cet. 
$\stackrel{Ð}{=}$

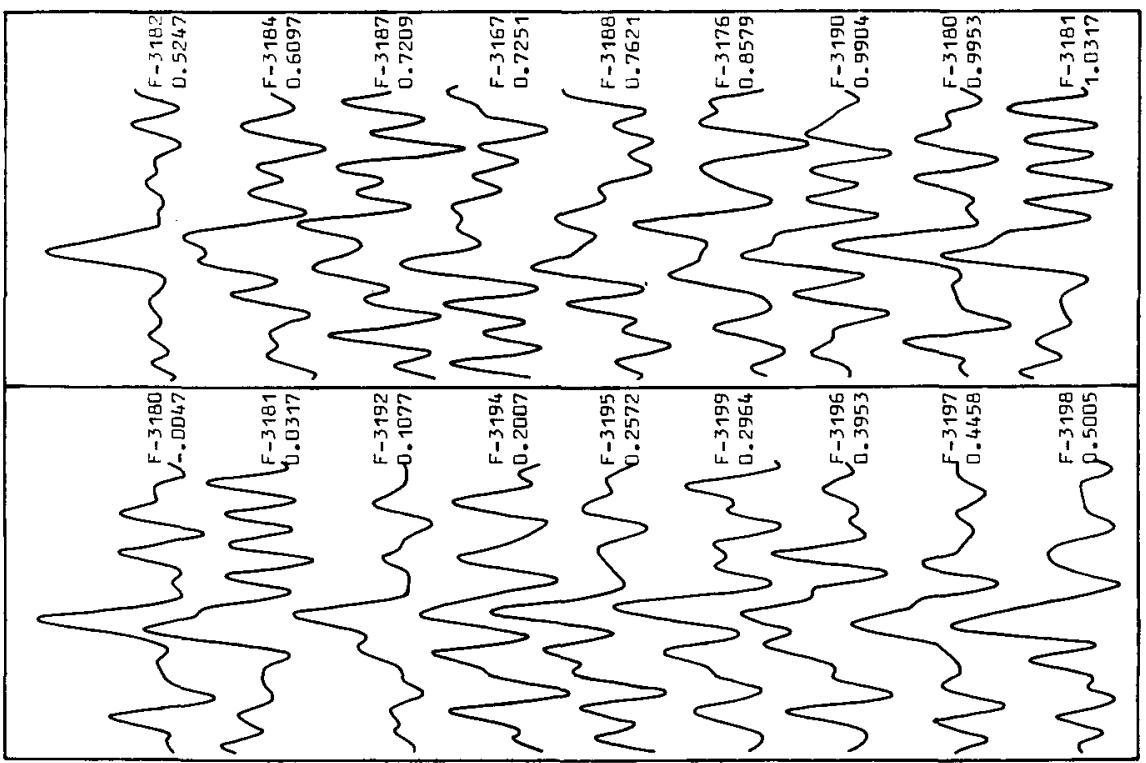

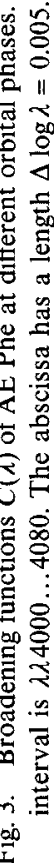
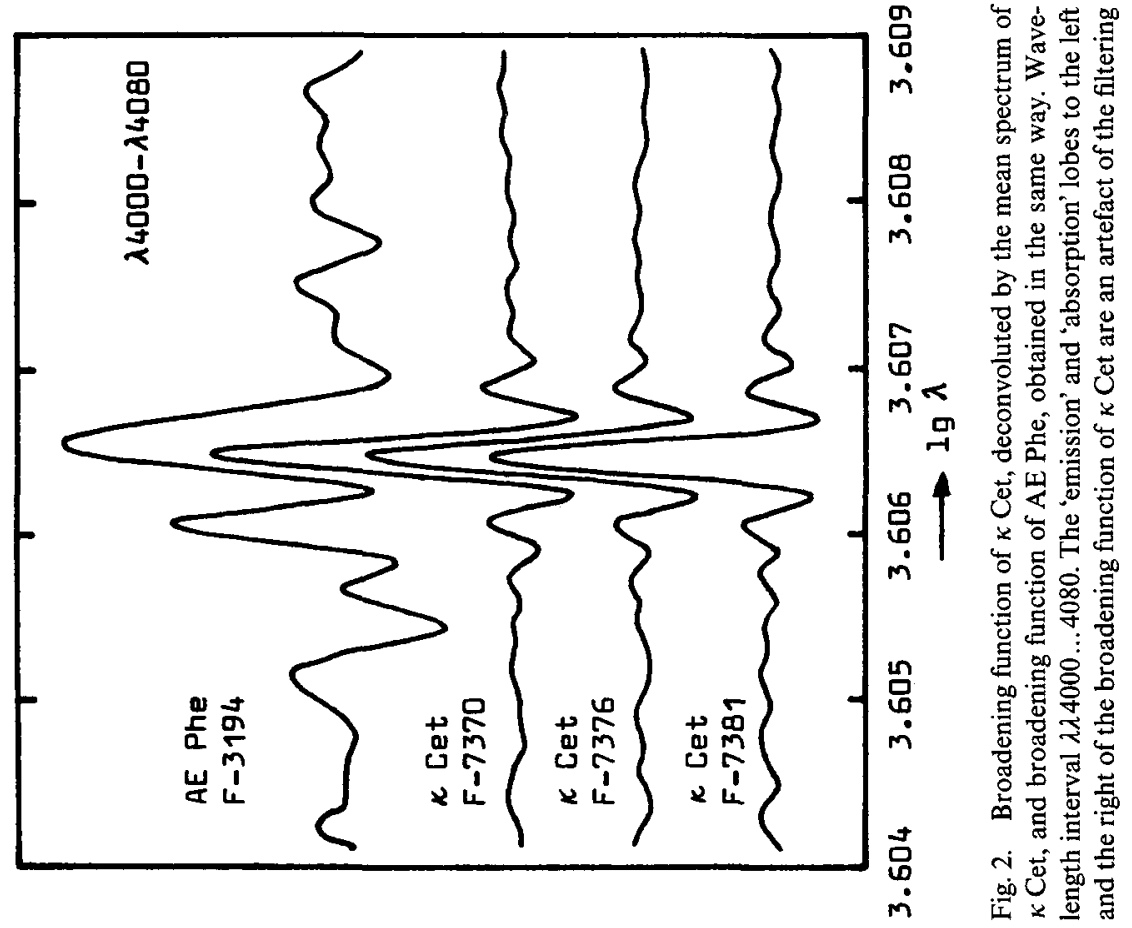


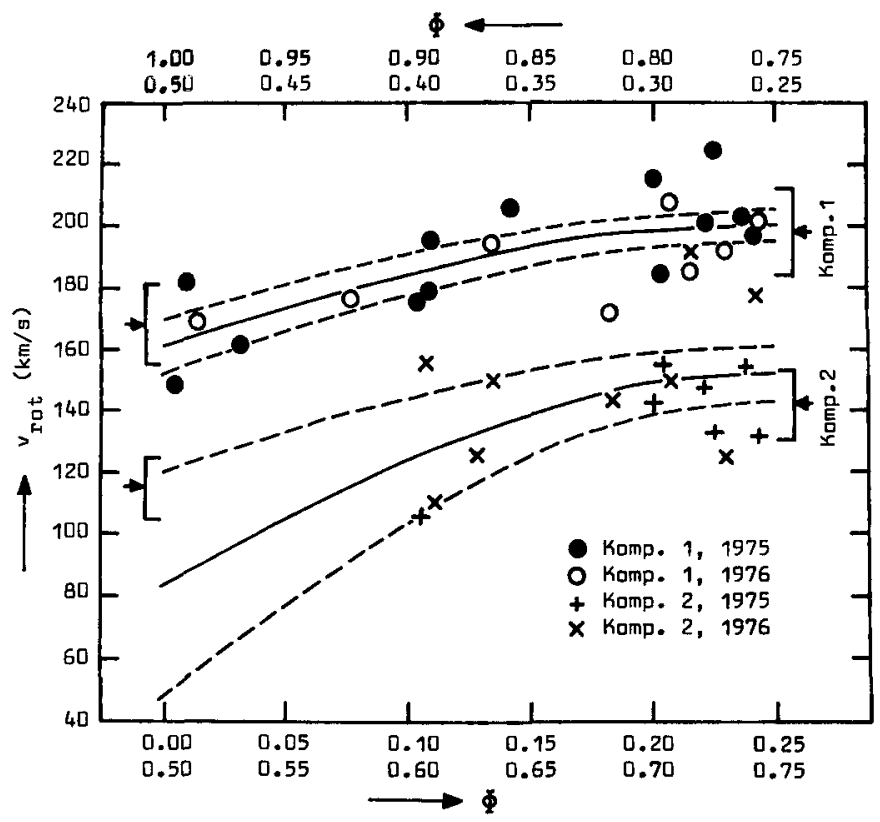

Fig. 4. Measured rotational velocities of the components of AE Phe derived from the widths of the broadening functions. The results (dots and crosses) agree well with the theoretical values, which are marked at the edges by arrows (and error bars). The theoretical profiles were calculated with the assumption of Roche lobe filling synchroneously rotating stars.
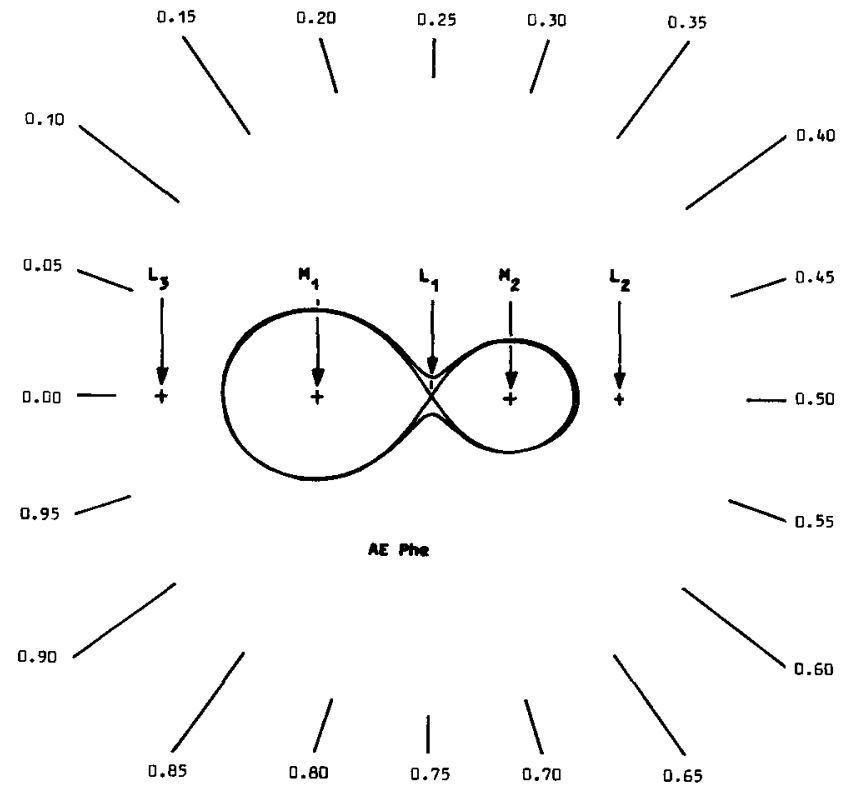

Fig. 5. Model of the system AE Phe. The mass centres of both stars and the Lagrangian points are indicated. 


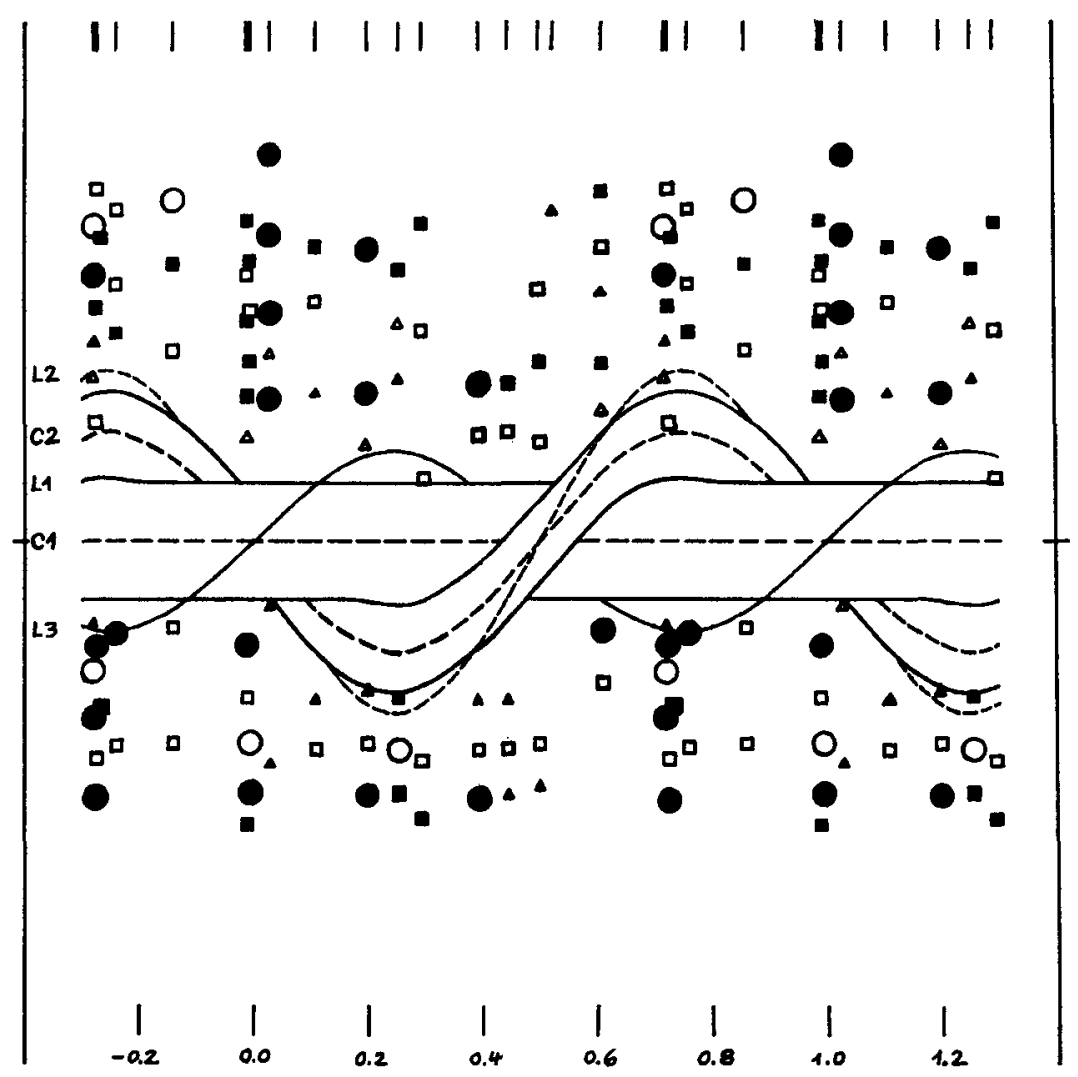

Fig. 6. The kinematics of special points of the system, as given in Figure 5, and referred to $M_{1}$, the mass centre of the primary component. Besides the two principal maxima of the broadening function, which indicate the motions of the two components, more maxima (absorption lines) and minima (emission lines) of the broadening function are indicated in Figure 4, with can hitherto not be explained (chromospheric lines?). These velocity components are marked in the figure as dots (absorption lines) and circles (emission lines). 